\title{
Binding Properties of CD94 to Sulfated Glycans and $\alpha 2,3-$ NeuAc- Containing Glycoproteins and Mutagenesis Analysis
}

\author{
Koji Higai*, Ayumi Itoh, Yuzo Imaizumi, Chiho Suzuki, Xin Xin, Yutaro Azuma and \\ Kojiro Matsumoto
}

Department of Clinical Chemistry, Faculty of Pharmaceutical Sciences, Toho University, Miyama 2-2-1, Funabashi, Chiba 247-8510, Japan

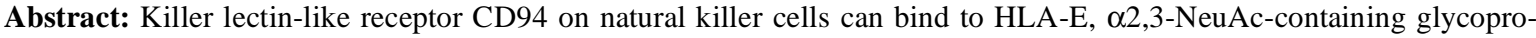
teins, and heparin. Using the glutathione-S-transferase-fused CD94 (rGST-CD94), we investigated its binding to plates coated with $\alpha 2,3-N e u A c-c o n t a i n i n g$ transferrin secreted by HepG2 cells (HepTF) and sulfate-containing glycanconjugated BSA. rGST-CD94 bound directly to several glycans with similar high affinities: rGST-CD94 bound to heparin-BSA, heparan sulfate-BSA, fucoidan-BSA, $\lambda$-carrageenan-BSA, and HepTF with Kd values of $36 \mathrm{nM}, 141 \mathrm{nM}, 36$ $\mathrm{nM}, 92 \mathrm{nM}$ and $84 \mathrm{nM}$, respectively. Different mutants of rGST-CD94 were generated by site-directed mutagenesis. Mutants N160A and C166G had reduced binding to sulfated glycans and HepTF, also F114A, L162A, D163A, and E164A showed reduced binding to sulfated glycans with some variability. These results indicated that CD94 interacts with sulfated glycans and $\alpha 2,3-\mathrm{NeuAc}$-containing glycoproteins suggesting that glycan binding sites in CD94 partially overlap with the HLA-E binding sites to modulate natural killer cell-dependent cytotoxicity.
\end{abstract}

Keywords: CD94, killer lectin-like receptors, heparin, sialy Lewis X, mutagenesis.

\section{INTRODUCTION}

Natural killer (NK) cells play important roles in innate immunity and in the immune surveillance of malignant transformed cells and viral-infected cells. The cytotoxic activity of NK cells is regulated by the balance of opposing signals transduced through activating and inhibiting cell surface receptors of the immunoglobulin (Ig)-like and C-type lectinlike receptors, and by signal integration and dynamic finetuning of NK cell responses [1,2].

Among C-type lectin-like receptors, CD94 forms disulfide-linked heterodimers with either natural killer group 2 (NKG2) A, B, C, E, or H [3-5]. The inhibitory receptors NKG2A and B have two immunoreceptor tyrosine-based inhibitory motifs (ITIMs) in their cytoplasmic domains, which associate with the $\mathrm{SH} 2$ domains of the protein tyrosine phosphatases SHP-1 and SHP-2 [6]. The activating receptors $\mathrm{NKG} 2 \mathrm{C}, \mathrm{E}$, and $\mathrm{H}$ have a positively charged $\mathrm{K}$ residue within their transmembrane regions, and associate with a negatively charged $\mathrm{D}$ residue in the disulfide-linked homodimer adaptor molecule DNAX-activating protein of 12 $\mathrm{kDa}$ (DAP12), which contains the immunoreceptor tyrosinebased activating motif (ITAM) [7]. In human cells, the ligand for CD94/NKG2A, B, C, and E is the non-classical major histocompatibility complex class (MHC)-1b molecule, human leukocyte antigen (HLA)-E, complexed with nonamer leader peptides of other MHC class 1 members, including HLA-A, B, C, and G [8-10].

*Address correspondence to this author at the Department of Clinical Chemistry, Faculty of Pharmaceutical Sciences, Toho University, Miyama 2-2-1, Funabashi, Chiba 247-8510, Japan; Tel: +81-474-72-1481; Fax: +81-4741485; E-mail: koji@phar.toho-u.ac.jp
The C-type lectin domain (CTLD) fold has a double-loop structure with its $\mathrm{N}$ - and $\mathrm{C}$-terminal $\beta$ strands ( $\beta 1$ and $\beta 2$ ) coming close together to form an antiparallel $\beta$-sheet. The most conserved CTLD residues (C1-C4) form disulfide bridges: $\mathrm{C} 1$ and $\mathrm{C} 4$ link $\beta 5$ and the $\alpha 1$ helix to form the whole domain loop, while $\mathrm{C} 2$ and $\mathrm{C} 3$ link $\beta 3$ and $\beta 5$ to form the long loop region that is involved in $\mathrm{Ca}^{2+}$-dependent carbohydrate binding. The C-type lectin-like receptor CD94 lacks one of two major $\alpha$ helices and most of the conserved $\mathrm{Ca}^{2+}$-binding residues present in other C-type lectins [11-13]. Their glycan ligands have yet to be elucidated.

Mouse melanoma B16-FI cells transfected with the fucosyltransferase (FUT)-3 gene and overexpressing sialyl Lewis $\mathrm{X}$ (sLeX) NeuAco2,3Galß1,4 (Fuc $\alpha 1,3)$ GlcNAc-R, in shorter N-glycans, are more susceptible to lysis by NK cells in vivo, which can be prevented by pretreatment with antiCD94 and anti-sLeX antibodies [14,15]. Similarly, it was found that FUT 3-transfected human erythroleukemia K562 (K562/FUT) cells selected for high expression of sLeX were more susceptible to lysis by human NK-derived KHYG cells in vitro, and that this susceptibility was suppressed by pretreatment of K562/FUT cells with anti-sLeX, and of KHYG cells with anti-NKG2D and anti-CD94 antibodies [16]. Using recombinant glutathione S-transferase (GST)-fused extracellular domains of NKG2D (AA 73-216) (rGSTNKG2D) and CD94 (AA 32-179) (rGST-CD94), and their site-directed mutants, it was found that rGST-NKG2D and rGST-CD94 can bind to plates coated with sLeX-expressing transferrin secreted by HepG2 cells (HepTF) [17] and heparin-conjugated bovine serum albumin (heparin-BSA) [18]. Moreover, it was found that rGST-NKG2A and rGSTNKG2C bind to heparin-BSA and HepTF with high binding affinities [19]. 
Table 1. Specific Primer Sets for Mutant Vectors

\begin{tabular}{|c|c|c|}
\hline Mutated Amino Acid & & Primer Sequences \\
\hline \multirow[t]{2}{*}{ F114A } & $\mathrm{F}$ & 5'-GCTTACTGGATTGGACTCTCTTACA-3' \\
\hline & $\mathrm{R}$ & 5'-TTGTTGACTGGAGCTCATAAAATC-3' \\
\hline N160A & $\mathrm{R}$ & 5'-GTTCTTTGTATTAAAAGTTTCAAATG-3' \\
\hline \multirow[t]{2}{*}{ L162A } & $\mathrm{F}$ & 5'-GCAGATGAATCCTGTGAAGATAAAAAT-3' \\
\hline & $\mathrm{R}$ & 5'-AGCATTTCCATTTGGATTATACGCT-3' \\
\hline D163A & $\mathrm{F}$ & 5'-GCTGAATCCTGTGAAGATAAAAATCG-3' \\
\hline E164A & $\mathrm{R}$ & 5'-ATCTAAAGCATTTCCATTTGGATTATACG-3' \\
\hline \multirow[t]{2}{*}{ C166G } & $\mathrm{F}$ & 5'- GGTGAAGATAAAAATCGTTATATCTGTA -3' \\
\hline & $\mathrm{R}$ & 5'- GGATTCATCTAAAGCATTTCCA -3' \\
\hline
\end{tabular}

In the present study, we further characterized the binding of rGST-CD94 and seven site-directed mutants to HepTF and sulfated glycans. We found that rGST-CD94 binds directly to heparin-BSA, heparan sulfate-BSA, fucoidan-BSA, $\lambda$ carrageenan-BSA, and HepTF with small $K_{d}$ values. Moreover, site-directed mutagenesis analysis revealed that N160 and C166 are essential for binding to HepTF and sulfated glycans, and that F114, L162, D163, and E164 contribute to sulfated glycan binding.

\section{MATERIALS AND METHODOLOGY}

\section{Cells and Cell Culture}

Human NK-derived KHYG cells from the Japanese Collection of Research Bioresources Cell Bank (JCRB) (Tokyo, Japan) were cultured in RPMI 1640 medium (Nissui Pharmaceutical Co., Tokyo, Japan) supplemented with $10 \%$ heatinactivated fetal bovine serum (FBS) (JRH Biosciences, Lenexa, KS), $0.6 \mathrm{mg} / \mathrm{ml} \mathrm{L-glutamate} \mathrm{(Wako} \mathrm{Chemicals} \mathrm{Co.,}$ Osaka, Japan), $100 \mathrm{U} / \mathrm{ml}$ penicillin (Banyu Pharmaceutical Co., Tokyo, Japan), and $100 \mu \mathrm{g} / \mathrm{ml}$ streptomycin (Meiji Seika Co., Tokyo, Japan) in a humidified atmosphere containing $5 \% \mathrm{CO}_{2}$ at $37{ }^{\circ} \mathrm{C}$. Recombinant interleukin-2 (Shionogi Pharmaceutical Co., Osaka, Japan) was added to the medium at a final concentration of $100 \mathrm{U} / \mathrm{ml}$. Human hepatoma HepG2 cells (JCRB) were cultured in 10\% FBS containing Dulbecco's modified Eagle's medium (DMEM) (Nissui).

\section{Preparation and Mutation of rGST-CD94 Constructs}

rGST-CD94, and its site-directed mutated constructs, were prepared as described previously $[17,18]$. Briefly, the extracellular domain of CD94 (coding for AA 32-179, pGEX4T-1/CD94) was amplified from KHYG-derived cDNA using 5'-TTGAAGCTATCCCACAAATTGA-3' (forward) and 5'-TTAAATGAGCTGTTGCTTACAG-3' (reverse). Site-directed mutant constructs of CD94, Q112A, F114A, N160A, L162A, D163A, E164A, and C166G were prepared using the KOD-plus-Mutagenesis kit (Toyobo, Tokyo, Japan) and pGEX4T-1/CD94 as the template according to the manufacturer's instructions. Primers designed for mutant vectors were shown in Table $\mathbf{1}$.

The purified PCR products were ligated into pGEX4T-1 vector (GE Healthcare Bio-Science, Uppsala, Sweden) with Ligation High (Toyobo). The recombinant plasmids were transformed into Chaperone competent cells, pTf16/BL21 (Takara, Otsu, Japan), and positive clones were confirmed by DNA sequencing.

After induction with $1 \mathrm{mM}$ isopropyl- $\beta$-Dthiogalactopyranoside (Promega Co., Madison, WI), the cells were harvested and sonicated for $10 \mathrm{~s} \times 6$ on ice. Recombinant proteins were purified on a GSTrap ${ }^{\mathrm{TM}} \mathrm{FF}$ column $(1 \mathrm{ml}$ packed volume; GE Healthcare) according to the manufacturer's instructions, and dialyzed against $1 \mathrm{mM}$ dithiothreitol (DTT)/PBS. Proteins were separated on $10 \%$ SDS-PAGE with and without pretreatment in 1\% 2-mercaptoethanol (2ME) at $95{ }^{\circ} \mathrm{C}$ for 3 min and stained with Coomassie brilliant blue. Protein concentrations were determined using the Advanced Protein Assay Reagent (Bio-Rad, Hercules, CA) with bovine serum albumin (BSA) as the standard.

\section{Preparation of Glycan-Coated Plates}

Glycans were conjugated with BSA in the presence of sodium cyanoborohydride and coated on 96-well plates according to a previous report [18]. Heparin (porcine intestinal mucosa; Wako), heparan sulfate (porcine intestinal mucosa; Funakoshi, Tokyo, Japan), fucoidan (marine algae; SigmaAldrich, St. Louis, MO), and $\lambda$-carrageenan (Kappaphycus cottonii; Wako) were conjugated with BSA and coated on 
the plates. Multimeric sLeX-expressing transferrin secreted by HepG2 cells (HepTF) [20] was purified from culture supernatant and directly coated on the plates [17]. As a control, plates were treated with $1 \% \mathrm{BSA} / \mathrm{PBS}$.

\section{Binding of rGST-CD94 to Glycan-BSA-Coated Plates}

To each glycan-coated plate, $100 \mu \mathrm{l}$ of 0 to $1.2 \mu \mathrm{M}$ rGST-CD94 in $20 \mathrm{mM}$ Tris- $\mathrm{HCl}$ buffer ( $\mathrm{pH}$ 7.4) containing $10 \mathrm{mM} \mathrm{CaCl}_{2}, 0.1 \% \mathrm{BSA}, 150 \mathrm{mM} \mathrm{NaCl}$, and $0.3 \%$ Tween (TBS-T) was added, and plates were incubated for $1 \mathrm{~h}$ at 37 ${ }^{\circ} \mathrm{C}$. After washing with TBS-T three times, the plates were further incubated with $100 \mu \mathrm{l}$ of $2 \mu \mathrm{g} / \mathrm{ml}$ peroxidase (POD)conjugated anti-GST antibody (Rockland Immunochemicals Inc., Gilbertsville, PA) in TBS-T for $1 \mathrm{~h}$ at room temperature. Then, after washing with TBS-T three times, the plates were incubated with $100 \mu \mathrm{l}$ of tetramethylbenzidine (TMB) solution (BioFX Lab., Owings Mills, MD) for $5 \mathrm{~min}$ at room temperature. The reaction was stopped by the addition of 100 $\mu \mathrm{l}$ of $1 \mathrm{M} \mathrm{H}_{2} \mathrm{SO}_{4}$, and absorbance was read at $450 \mathrm{~nm}$ with a Model DTX800 plate reader (Beckman Coulter, Fullerton, CA).

$K_{d}$ values of rGST-CD94 for glycans were determined using linear reciprocal plots $[21],[\mathrm{M}] / \Delta \mathrm{Abs}$ versus $[\mathrm{M}]$, where $[\mathrm{M}]$ is the concentration of rGST-CD94 (MW=43,784 Da). $K_{d}$ values were calculated from slopes $\left(1 / \mathrm{B}_{\max }\right)$ and $\mathrm{y}$ intercepts $\left(K_{d} / \mathrm{B}_{\max }\right)$, where $\mathrm{B}_{\max }$ is the maximum binding of rGST-CD94.

\section{Binding of Site-Directed Mutants of rGST-CD94 to Glycans}

To estimate the binding of site-directed mutants of rGST-CD94 to glycans, the concentrations of each mutant used were $22 \mathrm{nM}$ for heparin-BSA, fucoidan-BSA, $\lambda$ carrageenan-BSA, and HepTF, and $45 \mathrm{nM}$ for heparan sulfate-BSA. Binding was measured as described above and compared to wild-type rGST-CD94.

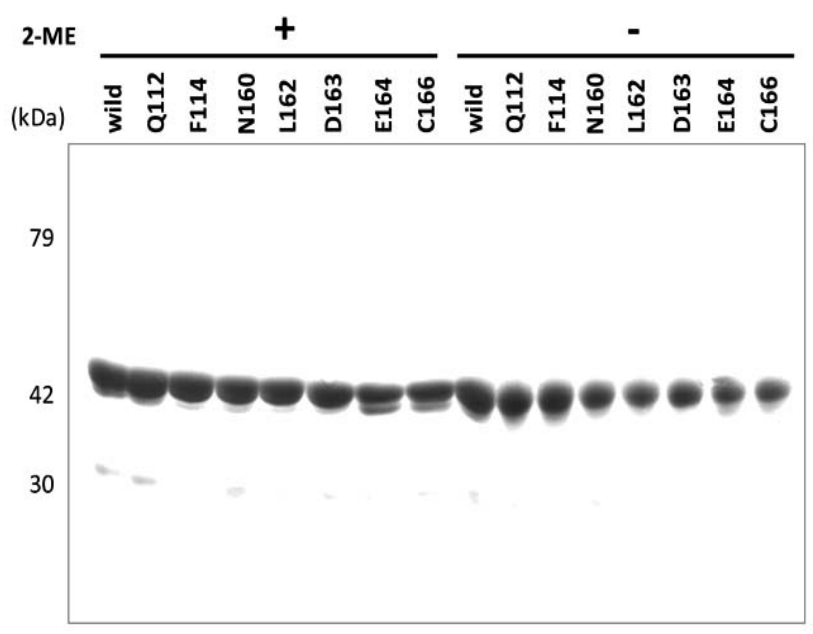

Fig. (1). SDS-PAGE for rGST-CD94 and its mutants.

rGST-CD94 and its mutants purified on a glutathione column and dialyzed against $1 \mathrm{mM}$ DTT/PBS were separated on 10\% SDSPAGE with (+; left lanes) and without (-; right lanes) pretreatment in $1 \% 2-\mathrm{ME}$ at $95^{\circ} \mathrm{C}$ for $3 \mathrm{~min}$. Bands at around $44 \mathrm{kDa}$ are rGSTCD94.

\section{RESULTS}

Binding of rGST-CD94 to Sulfate- and $\alpha 2,3-N e u A c-$ Containing Glycans

We prepared rGST-CD94 and its mutants using Chaperon competent cells, pTf16/BL21, proteins were purified on a glutathione-column. Recombinant proteins in $1 \mathrm{mM}$ DTT/PBS were separated on 10\% SDS-PAGE at around 44 $\mathrm{kDa}$, which was a similar size to those pretreated with $1 \% 2$ $\mathrm{ME}$, indicating that monomeric forms were obtained (Fig. 1). Chaperon proteins and GST were concomitantly detected at around 60 and $32 \mathrm{kDa}$, respectively.

We previously demonstrated that rGST-CD94 binds to $\alpha 2,3-\mathrm{NeuAc}$ on multi-antennary glycoproteins [17] and heparin-BSA [18]. Binding of rGST-CD94 to heparin-BSA is suppressed by soluble forms of heparin, heparan sulfate, dermatan sulfate, fucoidan, and $\lambda$-carrageenan [18]. To investigate the binding of CD94 to sulfated glycans and $\alpha 2,3$ NeuAc containing glycoprotein, we determined the binding of rGST-CD94 to plates coated with these glycans (Fig. 2). rGST-CD94 bound to heparin-BSA (Fig. 2A), heparan sulfate-BSA (Fig. 2B), fucoidan-BSA (Fig. 2C), $\lambda$-carrageenanBSA (Fig. 2D), and HepTF (Fig. 2E) in a dose-dependent manner.

Additionally, we determined their binding affinities $\left(K_{d}\right.$ values) using linear reciprocal plots for rGST-CD94 ranging from 10 to $210 \mathrm{nM},[\mathrm{M}] / \Delta \mathrm{Abs}$ versus [M] (Fig. 3). $K_{d}$ values of rGST-CD94 were $36 \mathrm{nM}\left(\mathrm{R}^{2}=0.997\right)$ for heparin-BSA (Fig. 3A), $141 \mathrm{nM}\left(\mathrm{R}^{2}=0.988\right)$ for heparan sulfate-BSA (Fig. 3B), $36 \mathrm{nM}\left(\mathrm{R}^{2}=0.993\right)$ for fucoidan-BSA (Fig. 3C), $92 \mathrm{nM}$ $\left(\mathrm{R}^{2}=0.985\right)$ for $\lambda$-carrageenan-BSA (Fig. 3D), and $84 \mathrm{nM}$ $\left(\mathrm{R}^{2}=0.972\right)$ for HepTF (Fig. 3E), indicating that rGST-CD94 bound to these glycans with similar high affinities. Although we tried to determined $\mathrm{K}_{\mathrm{d}}$ value for dermatan sulfate-BSA, the $K_{d}$ was not calculated by linear reciprocal plot analysis (data not shown).

In a previous report [18], we found that the binding of rGST-CD94 to heparin-BSA was not suppressed significantly by soluble forms of 2-O-, 6-O-, and $\mathrm{N}$-desulfated heparin at less than $100 \mu \mathrm{g} / \mathrm{ml}$. Here, we directly evaluated the binding of rGST-CD94 to 2-O-, 6-O-, and N-desulfated heparin-BSA-coated plates. Again, minimal binding of rGST-CD94 $(1.0 \mu \mathrm{M})$ to 2-O-, 6-O-, and N-desulfated heparin-BSA was detected (data not shown), suggesting that either 2-O-, 6-O-, or N-sulfate residues are necessary in heparin for binding to rGST-CD94.

\section{Mutagenesis Analysis for the Binding of rGST-CD94 to Glycans}

Residues Q112, F114, N160, L162, D163, and E164 in CD94 are essential for its recognition of HLA-E [22, 23]. Among these amino acids, F114 and N160 in CD94 are homologous to the NeuAc recognition domain of E-selectin [24], and also to the glycan-recognition domain of dectin-1 [25]. C166 in CD94 is essential for disulfide linkage formation between $\beta$-sheets [26]. We prepared rGST-CD94 mutants Q112A, F114A, N160A, L162A, D163A, E164A, and C166G, and compared their binding to wild-type rGSTCD94 to clarify the binding sites in CD94 for sulfated glycans and $\alpha 2,3-N e u A c-c o n t a i n i n g$ glycoproteins (Fig. 4). Mutagenesis analysis revealed that CD94 mutants N160A 


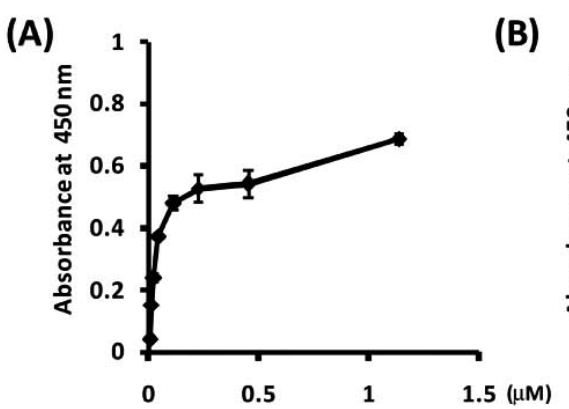

(C)

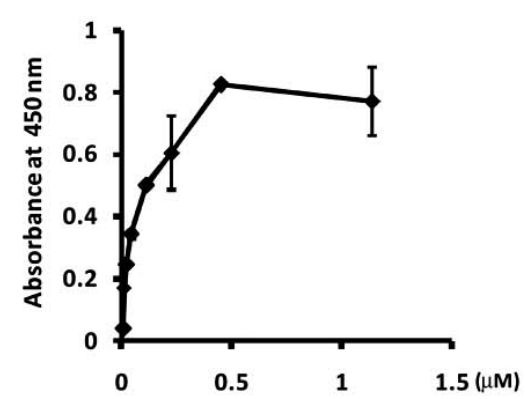

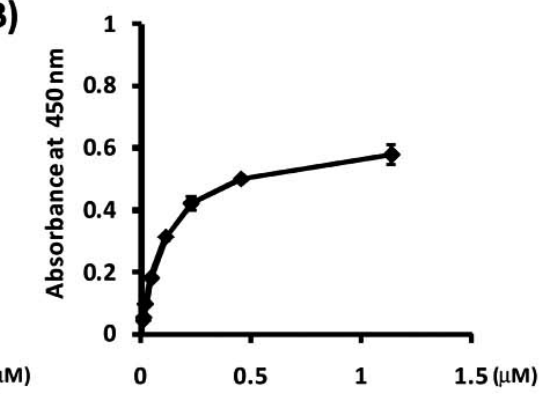

(D)

(E)

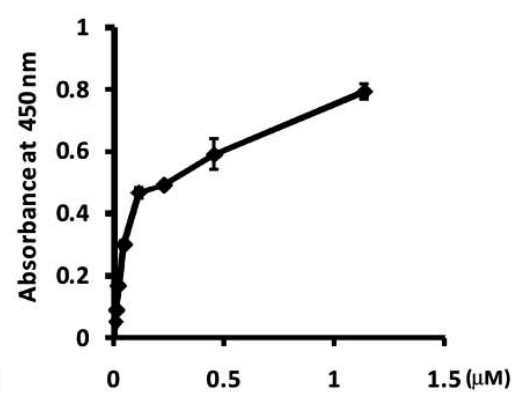

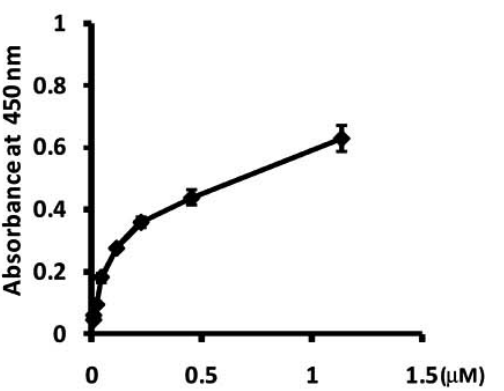

Fig. (2). Binding of rGST-CD94 to sulfated glycans and $\alpha 2,3-N e u A c$ containing glycoprotein.

rGST-CD94 (0 to $1.2 \mu \mathrm{M})$ was added to plates coated with (A) heparin-BSA, (B) heparan sulfate-BSA, (C) fucoidan-BSA, (D) $\lambda$ carrageenan-BSA, and (E) HepTF for $1 \mathrm{~h}$ at $37{ }^{\circ} \mathrm{C}$, and binding was determined using POD-conjugated anti-GST antibody. The results are given as means $\pm \mathrm{SD}(\mathrm{n}=3)$.

(A)

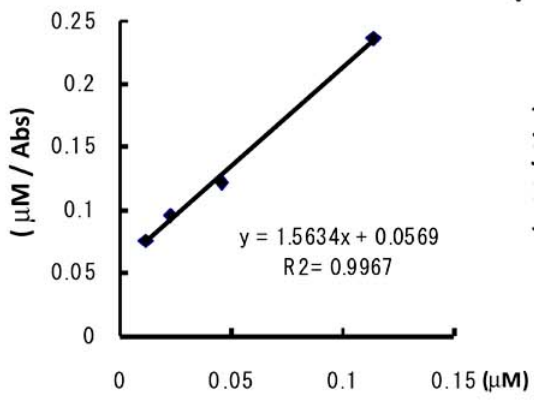

(C)

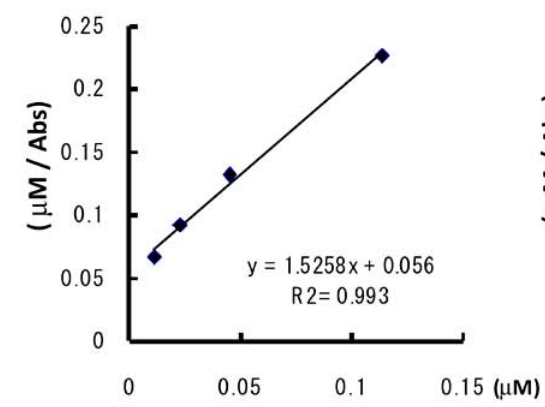

(B)

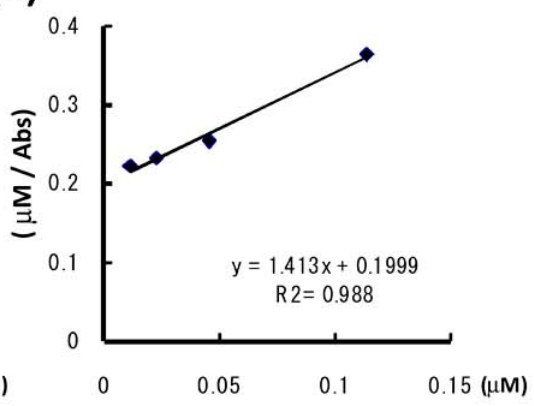

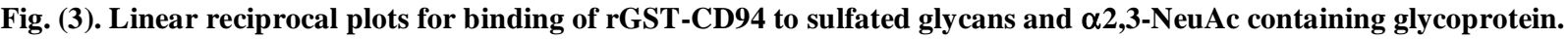

Linear reciprocal plots, [M]/AAbs vs. [M], for binding of rGST-CD94 to (A) heparin-BSA, (B) heparan sulfate-BSA, (C) fucoidan-BSA, (D) $\lambda$-carrageenan-BSA, and (E) HepTF as determined in Fig. (2). The results for binding of 11 to $220 \mathrm{nM}$ rGST-CD94 were used, and $\mathrm{K}_{\mathrm{d}}$ values were calculated from the slopes $\left(1 / \mathrm{B}_{\max }\right)$ and $\mathrm{y}$-intercepts $\left(\mathrm{K}_{\mathrm{d}} / \mathrm{B}_{\max }\right)$, where $[\mathrm{M}]$ and $\mathrm{B}_{\max }$ are the concentrations and maximum binding of rGST-CD94, respectively. 
and $\mathrm{C} 166 \mathrm{G}$ had reduced binding to heparin-BSA (Fig. 4A), heparan sulfate-BSA (Fig. 4B), fucoidan-BSA (Fig. 4C), $\lambda$ carrageenan-BSA (Fig. 4D), and HepTF (Fig. 4E), while the effects of F114A, L162A, D163A, and E164A on binding were insignificant in HepTF, and variable among sulfated glycans.

These results suggested that interactions between CD94 and glycans are partly composed of hydrophobic and/or hydrogen bonding interactions, and that the binding sites in

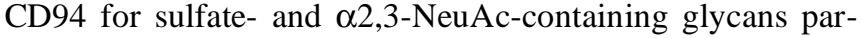
tially overlap with their protein ligand HLA-E binding sites.

\section{DISCUSSION}

In the present study, we found that the rGST-CD94 monomer binds directly to heparin-BSA $\left(K_{d}: 36 \mathrm{nM}\right)$, heparan sulfate-BSA (141 nM), fucoidan-BSA (36 nM), $\lambda$ carrageenan-BSA (92 nM), and HepTF (84 nM) with small $K_{d}$ values, and also to dermatan sulfate-BSA (value not determined). Previous competitive binding assays revealed that binding of rGST-CD94 to heparin-BSA is not suppressed by keratan sulfate, chondroitin sulfate $\mathrm{A}, \mathrm{B}$, and $\mathrm{C}$, hyaluronic acid, mannan, $\beta$-glucan, or by either 2-O-, 6-O-, or $\mathrm{N}-$ desulfated heparin [18]. We previously found that rGSTNKG2A and rGST-NKG2C also bind to heparin-BSA (20 and $40 \mathrm{nM}$ ), heparan sulfate-BSA (185 and $151 \mathrm{nM})$, fucoidan-BSA (1.35 and $0.27 \mathrm{nM}), \lambda$-carrageenan-BSA $(16.3$ and $6.7 \mathrm{nM}$ ), and HepTF (80 and $114 \mathrm{nM}$ ), respectively, and that 2-O-sulfate residues in heparin are essential for binding [19].

The binding affinities of CD94/NKG2x to HLAE/peptide complexes are peptide-dependent, and these interactions have very fast association and dissociation rate constants, although inhibitory receptor CD94/NKG2A binds to HLA-E/peptides with a much lower $K_{d}$ than stimulatory receptor CD94/NKG2C [27]. Inhibitory receptor CD94/ NKG2A and stimulatory receptor CD94/NKG2E display essentially identical affinities for HLA-E/peptide complexes with $K_{d}$ values ranging from 0.7 to $\sim 20 \mu \mathrm{M}$ for different peptides, which are uniformly 6-fold stronger than those of CD94/NKG2C, with $K_{d}$ values ranging from $\sim 4$ to $>100 \mu \mathrm{M}$ [28]. CD94, NKG2A, and NKG2C monomers bind to glycan ligands with higher affinities compared to these HLAE/peptide complexes, suggesting that these glycan ligands compete with protein ligands to modulate NK cell-dependent cytotoxicity.

The crystal structure of the CD94 homodimer revealed that CD94 is quite similar to the classical C-type lectin fold despite lacking one major $\alpha$-helix and $\mathrm{Ca}^{2+}$-binding loop [11]. Based on the structure of NKG2D/MICA, CD94/NKG2A should bind to HLA-E, with CD94 oriented over the $\alpha 1$ helix of HLA-E and the C-terminal part of the peptide, and NKG2A over the $\alpha 2$ helix [23]. A binding study using alanine substitutions of HLA-E loaded with the HLA-
(A)

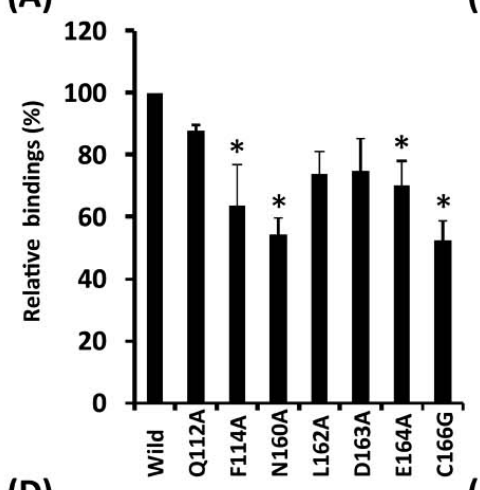

(D)

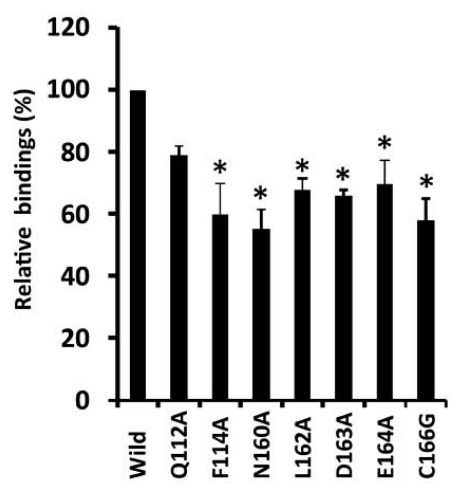

(B)

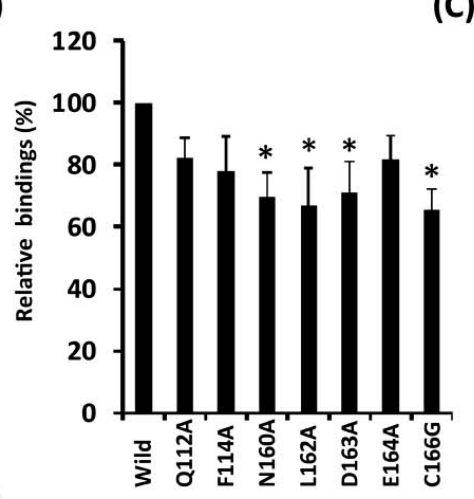

(E)

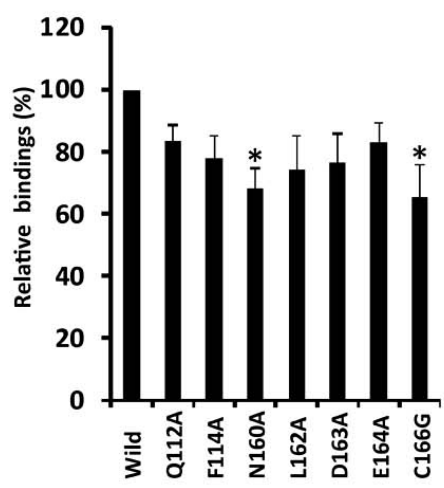

(C)

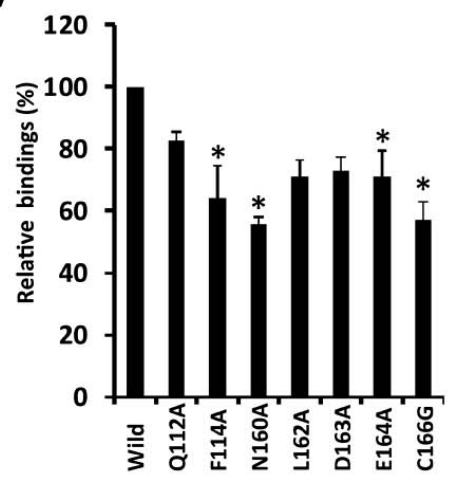

Fig. (4). Mutagenesis study for binding of rGST-CD94 to glycans.

The binding of rGST-CD94 mutants Q112A, F114A, N160A, L162A, D163A, E164A, and C166G to glycan-coated plates, including (A) heparin-BSA, (B) heparan sulfate-BSA, (C) fucoidan-BSA, (D) $\lambda$-carrageenan-BSA, and (E) HepTF, was determined as described in the legend of Fig. (2). The concentrations of rGST-CD94 wild-type and mutants used were $22 \mathrm{nM}$ for heparin-BSA, fucoidan-BSA, $\lambda$ carrageenan-BSA, and HepTF, and $45 \mathrm{nM}$ for heparan sulfate-BSA. The relative binding of rGST-CD94 mutants to each glycan is represented as $100 \%$ of wild-type rGST-CD94. The results are given as means \pm SD $(n=3)$, and asterisks denote significance differences $(P<0.05)$. 


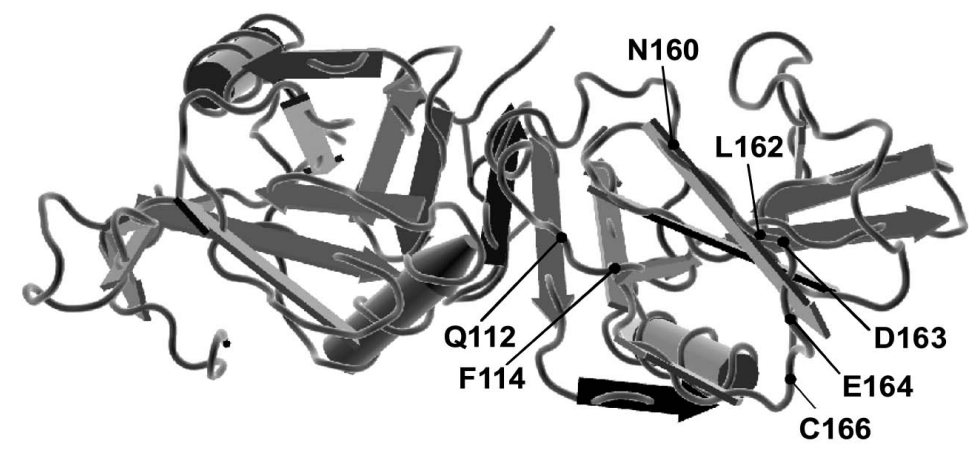

Fig. (5). Ribbon structure of CD94-NKG2A complex and mutated amino acids on CD94.

The structure of CD94-NKG2A complex was obtained from the Protein Data Base (PDB) \#3BDW.

B7 signal peptide, VMAPRTVLL, to CD94/NKG2 expressing cells showed that CD94/NKG2A and CD94/NKG2C bind to the top of $\alpha 1$ and $\alpha 2$ helices of the HLA-E heavy chain [29].

The crystal structure and alanine scanning have also revealed that loops 2,3 , and 5 , and $\beta$ strands 6 and 7 in CD94, interact broadly with residues $65-89$ in the $\alpha 1$ helix of HLAE, and that Q112, F114, N160, I162, D163, E164, and D168 in CD94, and Q212, R137, and R215 in NKG2A are important for the interaction. However, CD94 is positioned mostly over the $\alpha 1$-helix of HLA-E, and NKG2A over the $\alpha 2$-helix, and CD94 plays a crucial role in interacting with HLA-E while the role of NKG2A is more limited [23, 30]. The crystal structure of CD94/NKG2A with the HLA-E/peptide complex shows that two stretches of CD94 make significant contacts with the HLA-E platform: CD94 Q112, Q113, and F114 are directly adjacent to the interface with NKG2A; and CD94 N160, L162, D163, E164, F114, and L162 bind to a hydrophobic patch composed of HLA-E V76, E173, and F at $\mathrm{P} 8$ in the peptide [22]. The interaction between CD94/NKG2A and HLA-E is also driven by charged interactions: an acidic region of CD94 with a basic region on the $\alpha 1$ helix, and a basic region of NKG2A with the $\alpha 2$ helix of HLA-E [30]. The crystal structure of the CD94/NKG2A heterodimer indicated that the dimer interface is dominated by nonpolar interactions that comprise $\sim 2 / 3$ of the interface, and by two salt bridges: E161 and K135 in NKG2A salt-bridged to $\mathrm{K} 64$ and D106 in CD94, respectively [23]. F at P8 in the HLA-G leader sequence (VMAPRTLFL) is preferentially recognized by CD94/NKG2A and CD94/NKG2C. A hydrophobic patch on HLA-E composed of 173 and V76, and F at P8 in the peptide which interacts with CD94 L112 and F114, are critical for the interaction between CD94/NKG2 and HLA-E/peptide. Charged interactions between R75 and R79 in HLA-E, and D163 and E164 in CD94, are also important; however, CD94/NKG2A is particularly sensitive to minor changes in the peptide conformation [5, 23].

Amino acids mutated in the present study are shown in a ribbon structure of CD94 (Fig. 5). Mutagenesis analysis of the interaction between CD94 and glycan ligands revealed that binding of rGST-CD94 to sulfated glycans and $\alpha 2,3-$ NeuAc-containing glycoprotein was reduced significantly by substitutions of N160A and C166G in CD94, while F114A, L162A, D163A, and E164A affected binding to sulfated glycans with some variability, but not significantly to HepTF.
C166 forms a disulfide-linkage between $\mathrm{C} 2$ and $\mathrm{C} 3$ to stabilize the conformation of the long loop region. The $\mathrm{C} 166 \mathrm{G}$ mutation may disturb this conformation to suppress glycan binding. Q112 and F114 in the C-terminal region of loop 3, and N160, L162, D163, and E164 in the $\beta 6$-sheet, are important for HLA-E binding. The N160A mutation in CD94 suppressed binding to both HepTF and sulfated glycans, and L162A, D163A, and E164A mutations affected binding to sulfated glycans, indicating that glycan binding sites may overlap with HLA-E binding. Further study is needed to clarify the glycan binding site.

Heparan sulfate proteoglycans (HSPGs) are expressed ubiquitously on the surfaces of animal cells and in the extracellular matrix. Low molecular weight and modified heparin species are suggested to inhibit heparanase, mitogenic signaling through competition of binding of growth factors to their receptors, and metastasis by blocking selectinmediated interactions of tumor cells with platelets, leukocytes, and endothelium [31-33]. Heparanase is implicated in a variety of pathologies, such as tumor growth, angiogenesis, metastasis, inflammation, and glomerular diseases. Heparanase is over-expressed in a variety of malignant tumors and inflammation, and is linked to tumor growth and metastasis through release of growth factors and remodeling of extracellular matrix, suggesting it as a target for anticancer therapy $[34,35]$. Tumor metastasis is facilitated by complex formation of tumor cells with P-selectin on platelets and interactions with L-selectin. Heparin inhibits P-selectinmediated platelet/tumor cell interaction and metastasis in an L-selectin-dependent manner, but does not affect E-selectin. A heparin-derived tetrasaccharide, $\Delta \mathrm{UA} 2 \mathrm{~S} \alpha 1,4 \mathrm{GlcNS} 6 \mathrm{~S} \alpha 1$, 4IdoA2S $\alpha 1,4 \mathrm{GlcNS} 6 \mathrm{~S}$, strongly inhibits L- and P-selectin-Ig binding to sLeX-BSA [36]. GlcN6S in heparin is critical for interactions with P- and L-selectin [37]. P-selectin binds to heparin with a slow off-rate and a $K_{d}$ of $115 \mathrm{nM}$ according to surface plasmon resonance [38], and 1210 to $580 \mathrm{nM}$ according to quartz crystal microbalance [39].

Our results show that CD94 binds to heparin, heparan sulfate, fucoidan, $\lambda$-carrageenan, and HepTF $(\alpha 2,3$-NeuAc on multimeric $\mathrm{N}$-glycans), and suggest that the binding sites for these glycans partially overlap with those for protein ligands to modulate NK-dependent cytotoxicity. Further study is needed to clarify the role of glycans in cancer immunity. 


\section{ACKNOWLEDGEMENTS}

This research was partially supported by the Ministry of Education, Science, Sports and Culture, Grant-in-Aid for Young Scientists (B), 21790544, 2009, and by the "Open Research Center" Project.

\section{REFERENCES}

[1] Lanier LL. NK cell recognition. Annu Rev Immunol 2002; 23: 22574.

[2] Bryceson YT, Long EO. Line of attack: NK cell specificity and integration of signals. Curr Opin Immunol 2008; 20: 344-52.

[3] Hoare HL, Sullivan LC, Clements CS, et al. Subtle changes in peptide conformation profoundly affect recognition of the nonclassical MHC class I molecule HLA-E by the CD94-NKG2 natural killer cell receptors. J Mol Biol 2008; 377: 1297-303.

[4] Deng L, Mariuzza RA. Structural basis for recognition of MHC and MHC-like ligands by natural killer cell receptors. Semin Immunol 2006; 18: 159-66.

[5] Sullivan LC, Clements CS, Rossjohn J, Brooks AG. The major histocompatibility complex class Ib molecule HLA-E at the interface between innate and adaptive immunity. Tissue Antigens 2008; 72: 415-24.

[6] Le Dréan E, Vély F, Olcese L, et al. Inhibition of antigen-induced $\mathrm{T}$ cell response and antibody-induced NK cell cytotoxicity by NKG2A: association of NKG2A with SHP-1 and SHP-2 proteintyrosine phosphatases. Eur J Immunol 1998; 28: 264-76.

[7] Lanier LL, Corliss B, Wu J, Phillips JH. Association of DAP12 with activating CD94/NKG2C NK cell receptors. Immunity 1998; 8: 693-701.

[8] Lee N, Llano M, Carretero M, et al. HLA-E is a major ligand for the natural killer inhibitory receptor CD94/NKG2A. Proc Natl Acad Sci USA 1998; 95: 5199-204.

[9] Braud VM, Allan DS, O'Callaghan CA, et al. HLA-E binds to natural killer cell receptors CD94/NKG2A, B and C. Nature 1998; 391: 795-9.

[10] Borrego F, Ulbrecht M, Weiss EH, Coligan JE, Brooks AG. Recognition of human histocompatibility leukocyte antigen (HLA)-E complexed with HLA class I signal sequence-derived peptides by CD94/NKG2 confers protection from natural killer cell-mediated lysis. J Exp Med 1998; 187: 813-8.

[11] Boyington JC, Riaz AN, Patamawenu A, Coligan JE, Brooks AG, Sun PD. Structure of CD94 reveals a novel C-type lectin fold: implications for the NK cell-associated CD94/NKG2 receptors. Immunity 1999; 10: 75-82.

[12] Strong RK, McFarland BJ, NKG2D and Related Immunoreceptors. Adv Protein Chem 2004; 68: 281-312.

[13] Zelensky AN, Gready JE. The C-type lectin-like domain superfamily. FEBS J 2005; 272: 6179-217.

[14] Ohyama C, Tsuboi S, Fukuda M. Dual roles of sialyl Lewis X oligosaccharides in tumor metastasis and rejection by natural killer cells. EMBO J 1999; 18: 1516-25.

[15] Ohyama C, Kanto S, Kato K, et al. Natural killer cells attack tumor cells expressing high levels of sialyl Lewis $\mathrm{x}$ oligosaccharides. Proc Natl Acad Sci USA 2002; 99: 13789-94.

[16] Higai K, Ichikawa A, Matsumoto K. Binding of sialyl Lewis X antigen to lectin-like receptors on NK cells induces cytotoxicity and tyrosine phosphorylation of a $17-\mathrm{kDa}$ protein. Biochim Biophys Acta 2006; 1760: 1355-63

[17] Imaizumi Y, Higai K, Suzuki C, Azuma Y, Matsumoto K. NKG2D and CD94 bind to multimeric $\alpha 2,3$-linked $\mathrm{N}$-acetylneuraminic acid. Biochem Biophys Res Commun 2009; 382: 604-8.

[18] Higai K, Imaizumi Y, Suzuki C, Azuma Y, Matsumoto K. NKG2D and CD94 bind to heparin and sulfate-containing polysaccharides. Biochem Biophys Res Commun 2009; 386: 709-14.

[19] Xin X, Higai K, Imaizumi Y, et al. NKG2A and NKG2C bind to sulfated glycans and $\alpha 2,3$-NeuAc-containing glycoproteins. Biol Pharm Bull 2011; 34(4): 480-5.
[20] Higai K, Shibukawa K, Muto S, Matsumoto K. Targeted proteoglycomics analysis of sialyl Lewis $\mathrm{X}$ antigen expressing glycoproteins secreted by human hepatoma cell line. Anal Sci 2003; 19: 8592.

[21] Higai K, Suzuki C, Imaizumi Y, Xin X, Azuma Y, Matsumoto K. Binding Affinities of NKG2D and CD94 to Sialyl Lewis $\mathrm{X}$ Expressing N-Glycans and Heparin. Biol Pharm Bull 2011; 34: 8-12

[22] Kaiser BK, Pizarro JC, Kerns J, Strong RK. Structural basis for NKG2A/CD94 recognition of HLA-E. Proc Natl Natl Acad Sci USA 2008; 105: 6696-701.

[23] Sullivan LC, Clements CS, Beddoe T, et al. The heterodimeric assembly of the CD94-NKG2 receptor family and implications for human leukocyte antigen-E recognition. Immunity 2007; 27: 90011 .

[24] Somers WS, Tang J, Shaw GD, Camphausen RT. Insights into the molecular basis of leukocyte tethering and rolling revealed by structures of P- and E-selectin bound to SLe(X) and PSGL-1. Cell 2000; 103: 467-79.

[25] Brown J, O'Callaghan CA, Marshall AS, et al. Structure of the fungal $\beta$-glucan-binding immune receptor dectin-1: implications for function. Protein Sci 2007; 16: 1042-52.

[26] Zelensky AN, Gready JE. Comparative analysis of structural properties of the C-type-lectin-like domain (CTLD). Proteins 2003; 52: 466-77.

[27] Valés-Gómez M, Reyburn HT, Erskine RA, López-Botet M, Strominger JL. Kinetics and peptide dependency of the binding of the inhibitory NK receptor CD94/NKG2-A and the activating receptor CD94/NKG2-C to HLA-E. EMBO J 1999; 18: 4250-60.

[28] Kaiser BK, Barahmand-Pour F, Paulsene W, Medley S, Geraghty DE, Strong RK. Interactions between NKG2x immunoreceptors and HLA-E ligands display overlapping affinities and thermodynamics. J Immunol 2005; 174: 2878-84.

[29] Wada H, Matsumoto N, Maenaka K, Suzuki K, Yamamoto K. The inhibitory NK cell receptor CD94/NKG2A and the activating receptor CD94/NKG2C bind the top of HLA-E through mostly shared but partly distinct sets of HLA-E residues. Eur J Immunol 2004; 34: 81-90.

[30] Petrie EJ, Clements CS, Lin J, et al. CD94-NKG2A recognition of human leukocyte antigen (HLA)-E bound to an HLA class I leader sequence. J Exp Med 2008; 205: 725-35.

[31] Niers TM, Klerk CP, DiNisio M, et al. Mechanisms of heparin induced anti-cancer activity in experimental cancer models. Crit Rev Oncol Hematol 2007; 61: 195-207.

[32] Casu B, Vlodavsky I, Sanderson RD. Non-anticoagulant heparins and inhibition of cancer. Pathophysiol Haemost Thromb 2008; 36 : 195-203.

[33] Mousa SA, Petersen LJ. Anti-cancer properties of low-molecularweight heparin: preclinical evidence. Thromb Haemost 2009; 102 258-67.

[34] Nasser NJ. Heparanase involvement in physiology and disease. Cell Mol Life Sci 2008; 65: 1706-15.

[35] McKenzie EA. Heparanase: a target for drug discovery in cancer and inflammation. Br J Pharmacol 2007; 151: 1-14.

[36] Wei M, Tai G, Gao Y, et al. Modified heparin inhibits P-selectinmediated cell adhesion of human colon carcinoma cells to immobilized platelets under dynamic flow conditions. J Biol Chem 2004; 279: 29202-10

[37] Wang L, Brown JR, Varki A, Esko JD. Heparin's antiinflammatory effects require glucosamine 6-O-sulfation and are mediated by blockade of L- and P-selectins. J Clin Invest 2002; 110: $127-36$.

[38] Wang JG, Geng JG. Affinity and kinetics of P-selectin binding to heparin. Thromb Haemost 2003; 90: 309-16.

[39] Simonis D, Fritzsche J, Alban S, Bendas G. Kinetic analysis of heparin and glucan sulfates binding to P-selectin and its impact on the general understanding of selectin inhibition. Biochemistry 2007; 46: 6156-64. 